\title{
A new method for quantification of qualitative expectations
}

\author{
Joachim Zuckarelli*
}

Munich, Germany

Received: 1 August 2015

Revised: 7 November 2015

Accepted: 10 November 2015

\begin{abstract}
For many research purposes it is necessary to quantify qualitative inflation or other expectations from consumer or business surveys. The standard quantification method that is widely referred to in the literature for qualitative inflation expectations is the Carlson-Parkin method, with various extensions. This study proposes a novel quantification method that connects the survey respondents' inflation experience with forward looking information. The article outlines the new approach and applies it exemplarily to qualitative inflation survey data for the Euro area and the United States.
\end{abstract}

Keywords: quantification, quantification methods, qualitative surveys, qualitative expectations, inflation expectations

JEL Classification Codes: C42, D84, E31

\section{Introduction}

Surveys often ask respondents for qualitative answers even though the variable that is surveyed is actually quantitative in nature. Surveys asking respondents to assess the likely course of inflation over a given period of time are typical for this kind of survey design. Instead of asking directly for a numerical value, these surveys let respondents predict whether the rate of inflation will go up, go down or stay the same. For practical purposes, however, it is often necessary to have quantitative data on people's expectations. Therefore, several methods have been proposed in the literature to quantify qualitative survey answers, starting with Anderson (1952) and Theil (1952). Today, two principal quantification approaches can be distinguished: the regression approach developed by Pesaran (1984), and the probability method significantly brought forward by Carlson/Parkin (1975). The latter method is the most commonly used

\footnotetext{
*E-mail: joachim@zuckarelli.de.
}

Citation: Zuckarelli, J. (2015) A new method for quantification of qualitative expectations, Economics and Business Letters, 4(3), 123-128. 
quantification approach. It has undergone several extensions and improvements; for example, by Henzel/Wollmershäuser (2005).

One feature of Carlson/Parkin's (1975) original approach is that it calibrates the mean of the quantified expectations to be equal to the mean of the actual realizations of the variable in question. This calibration assumption, which also brings about problems when testing the unbiasedness of expectations, is difficult to maintain since in reality survey respondents obviously do not know the future course of the variable in question at the time they form expectations.

However, such assumptions are an inherent and unavoidable part of any quantification method. This paper proposes a new approach in which the assumptions neither require survey respondents to know the future course of the surveyed variable nor impose unbiasedness on the quantified expectations.

The structure of the paper is as follows: Section 2 discusses briefly some important theoretical aspects of quantification before Section 3 explains the new quantification approach. Section 4 provides an empirical example and shows the new method at work. Section 5 concludes.

\section{Why quantification and why different methods?}

Before discussing the new quantification method in detail it is worth addressing the legitimate question why qualitative surveys are conducted at all. An obvious reason is that asking people qualitative instead of quantitative questions makes it easier for them to answer and thus increases the likelihood of their participation in the survey. Furthermore, Pesaran (1984) argues that when answering surveys people do not have point forecasts in mind but rather expectations that might be best described as 'attitudes' or 'states of mind'. Therefore, qualitative surveys are better fitted to people's mental processes and are thus less prone to measurement errors.

However, quantification implicitly assumes that people either have a quantitative idea of the variable that is surveyed or would (when asked) come to that value in a way similar to the one underlying the quantification method. Otherwise, the quantification approach would just be a function transforming respondents' real (qualitative) expectations into a quantitative forecast that is no longer related to their actual expectations. The result would be a new forecast to which the survey responses are simply an input, albeit an important one.

Qualitative data are on a lower scale level (ordinal) than quantitative data (ratio); hence, they are not sufficient for calculating quantitative figures. This implies that all quantification methods must add information to the original qualitative survey data. If this information is to reflect what survey respondents might have had in mind when thinking about their answers, the methods have to be based on assumptions about how people actually form expectations. However, the process of expectation formation cannot be directly observed. Therefore, all testing of hypotheses on the basis of quantified survey results actually means testing two hypotheses simultaneously (at least as long as no direct quantitative surveys are available): first, the actual hypothesis under consideration (e.g., the unbiasedness of inflation expectations); and, second, the expectation formation hypothesis assumed by the quantification method. Thus, a hypothesis that is actually true may be rejected by statistical tests because the quantification approach does not properly reflect the expectation formation process of the survey respondents. Accordingly, it is critical to correctly model respondents' expectation formation. This in turn implies that we must allow for different types of expectation formation assumptions because people or groups of people (e.g., bankers, consumers, and economists) may differ in how they produce expectations. This is what actually constitutes a right to exist for very different quantification approaches, far beyond the technical aspects of the calculation. 


\section{A new quantification method based on conditional expectations}

It is natural to assume that in forming inflation expectations people also rely on experiences made earlier. Based on a modified adaptive learning model Malmedier/Nagel (2015) and Madeira/Zafar (2014) show empirically that inflation expectations depend on inflation experience and that expectations differ between age cohorts due to differences in inflation experiences that individuals have made over the course of their life. In the quantification approach presented in this paper individuals make use of their experience by forming expectations based on the distribution of past inflation.

Let us assume that survey participants believe at time $t$ that the value of the surveyed variable (say, inflation) at time $t+k$ will a priori follow the same distribution that it showed empirically over time in the past. Now, survey respondents form a qualitative expectation (inflation will increase, decrease or stay the same) using the information available to them. Lacking any better information, to come to a quantitative assessment, they simply expect inflation of time $t+k$ to be the mean of the past inflation distribution conditional on their qualitative assessment.

More formally: Survey participant $i$ forms at time $t$ a qualitative expectation over the rate of inflation in $t+k$. We will describe this qualitative expectation with two binary variables: $d_{i}^{u p}$ takes the value of 1 if individual $i$ expects inflation to increase and 0 otherwise, $d_{i}^{\text {down }}$ equals 1 if and only if individual $i$ expects a decrease in inflation:

$$
\begin{aligned}
d_{i}^{u p} & =\left\{\begin{array}{l}
1 \text { if } \pi_{i t+k}^{e}>\pi_{t} \\
0 \text { if } \pi_{i t+k}^{e} \leq \pi_{t}
\end{array}\right. \\
d_{i}^{\text {down }} & =\left\{\begin{array}{l}
1 \text { if } \pi_{i t+k}^{e}<\pi_{t} \\
0 \text { if } \pi_{i t+k}^{e} \geq \pi_{t}
\end{array}\right.
\end{aligned}
$$

where $\pi_{i t+k}^{e}$ is individual $i$ 's expected inflation rate for time $t+k$ and $\pi_{t}$ is actual inflation at time $t$. Each individual $i$ has an experience horizon of $n_{i}$, that means he/she knows past inflation over the period $\left[t-n_{i}, t\right]$. Individuals assume $a$ priori that the possible inflation rates at time $t+k$ follow the distribution $F_{i}(x)$ which is the empirical distribution of inflation over the period $\left[t-n_{i}, t\right]{ }^{1}$

Using this a priori distribution and his/her qualitative expectations $d_{i}$ individual $i$ derives the quantitative expectation simply as the mean of the historical distribution conditional on an inflation increase or decrease, depending on his/her qualitative expectation:

$$
\pi_{i t+k}^{e}=d_{i}^{u p} \cdot \bar{F}_{i}\left(x \mid x>\pi_{t}\right)+\left(1-d_{i}^{u p}-d_{i}^{\text {down }}\right) \cdot \pi_{t}+d_{i}^{\text {down }} \cdot \bar{F}_{i}\left(x \mid x<\pi_{t}\right)
$$

where $\bar{F}_{i}(x)$ is the mean of the empirical distribution function $F_{i}(x)$. For sake of simplicity it is assumed that $n_{i}=n \forall i$, meaning that all survey respondents have the same experience horizon. Consequently, the empirical distribution of past inflation rates covering the period $[t-n, t]$ is $F(x)$ for all individuals.

With $\hat{A}_{t}, \hat{B}_{t}$ and $\hat{C}_{t}$ being the proportion of respondents expecting inflation to rise, stay the same and decrease, respectively, the estimator for the population's mean inflation expectation can be calculated as:

$$
\pi_{t+k}^{e}=\hat{A}_{t} \cdot \bar{F}\left(x \mid x>\pi_{t}\right)+\hat{B}_{t} \cdot \pi_{t}+\hat{C}_{t} \cdot \bar{F}\left(x \mid x<\pi_{t}\right)
$$

One special case needs to be treated separately: Whenever $\pi_{t}=\max \left(\pi_{t-n}, \ldots, \pi_{t}\right)$ or $\pi_{t}=$ $\min \left(\pi_{t-n}, \ldots, \pi_{t}\right)$ (i.e. when inflation at the time of expectation formation is at the boundary of the inflation range of the experience horizon timeframe) and the qualitative expectation of

\footnotetext{
${ }^{1}$ The empirical distribution $F_{i}(x)$ is just the fraction of periods in $\left[t-n_{i}, t\right]$ with $\pi_{t}<x$ to the total number of periods covered by the expericence horizon, i.e. $t-n_{i}+1$.
} 
individual $i$ is that inflation will further rise (further fall), it is assumed that the expected absolute change in inflation $\Delta \pi_{i t+k}^{e}$ equals the average increase (decrease) of inflation over the experience horizon timeframe given that inflation actually increased (decreased). Formally, for the case $\pi_{t}=\max \left(\pi_{t-n}, \ldots, \pi_{t}\right)$ and a qualitative expectation of increasing inflation:

$$
\Delta \pi_{i t+k}^{e}=\frac{1}{|Z|} \sum_{z \in Z}\left(\pi_{z}-\pi_{z-1}\right)
$$

with $Z=\left\{z \in\{t-k+1, \ldots, t\} \mid \pi_{z}-\pi_{z-1}>0\right\}$.

The core assumption of this new quantification approach is that individuals a priori expect that the possible inflation rates at time $t+k$ are distributed in the same way as actual inflation was distributed over the period defined by their experience horizon. ${ }^{2}$ This might be considered a very strong assumption. However, it seems still more realistic than making inflation expectations depend on future inflation realizations as the original approach of Carlson/Parkin (1975) does. The proposed method only makes use of information potentially available to survey respondents at the time of expectation formation. Also, even though it employs past inflation data the new approach is not simply adaptive, since the qualitative assessment incorporates forward-looking information. How exactly this information is processed by the respondents does not need to be modeled. This allows to account for very heterogenous expectation formation processes. Only their results, the qualitative expectations, are fed into the quantification method. Finally, the proposed approach does not require any assumption that makes meaningful statistical tests of important expectation characteristics like unbiasedness virtually impossible.

The equality of the experience horizon across individuals is an assumption that keeps the whole construct computable. Carlson/Parkin (1975) use a similar assumption when assuming 'indifference limens' to be the same across individuals and constant over time. Nevertheless, the experience horizon must be chosen by the researcher. In any case, to ensure robustness it is recommended to work with several different horizons when testing hypotheses based on quantified expectations.

\section{Application of the new quantification method}

In this section the new quantification method is exemplarily applied to qualitative data from the Financial Market Report, a publication of the Centre for European Economic Research at the University of Mannheim, Germany. The Financial Market Report is based on a monthly survey among analysts from banks, insurance companies and large industrial companies. The survey participants are asked to assess whether inflation will be higher, lower or at the same level in six months compared to today.

For this example application of the new quantification approach, survey data from January 2003 to August 2014 is used. The parameter $n$, the experience horizon of survey participants, is set to 48 months, meaning that respondents consider the inflation experience of four years when forming their inflation expectations.

The results for the Euro area and the United States are depicted in Figure 1. The figure also shows the results of the traditional Carlson-Parkin approach. Mean absolute errors of expectations quantified with the new method are slightly lower than those of the Carlson-Parkin expectations (1.01 percentage points vs. 1.12 percentage points for US inflation; 0.51

\footnotetext{
${ }^{2}$ The moving fixed-length experience horizon could be easily adapted to become a fixed-start experience horizon considering the full lifetime inflation experience of the respondents. The fixed-length specification used in this paper is consistent with the idea that older experiences fade out over time.
} 
percentage points vs. 0.79 percentage points for Euro Area inflation). Likewise, the correlation between quantified expectations and actual inflation is higher with the new approach than with the traditional Carlson-Parkin method ( 0.38 vs. 0.33 for the United States; 0.62 vs. 0.27 for the Euro area).

The experience horizon of four years might be considered short compared to Malmedier/Nagel's (2015) 'lifetime experience'. However, it seems justified to assume that professionals who deal with inflation on a day-to-day basis focus more on recent events than on experiences made earlier in their life. In general, the more is known about the respondents the more can the experience horizons be tailored to their situation.

Figure 1. Quantified expectations.
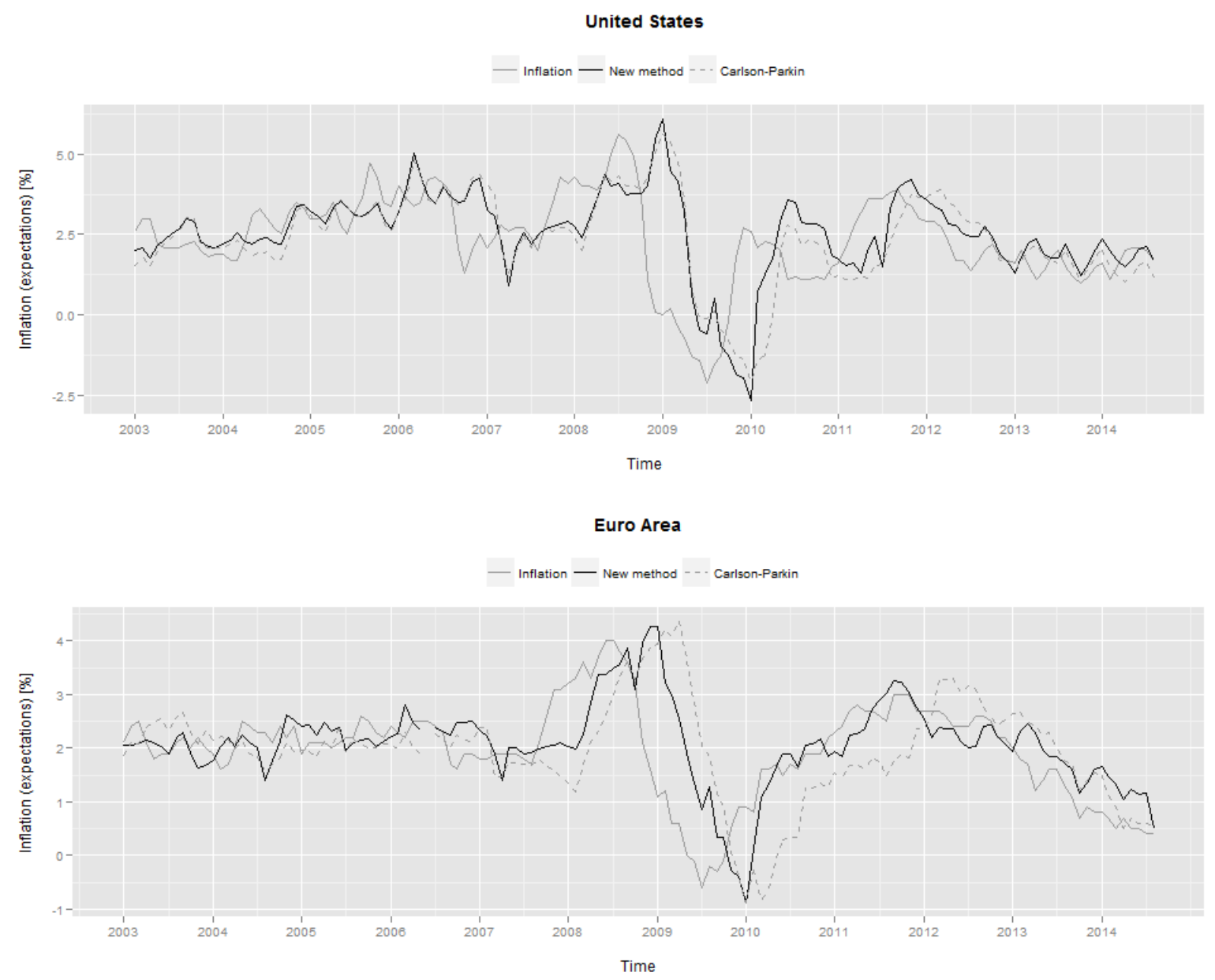

\section{Conclusion}

This article presents a new method for the quantification of qualitative survey data as an alternative to the widely-used Carlson-Parkin method. It is based on the idea that in forming expectations survey respondents connect their inflation experience with forward-looking information. Unlike the Carlson-Parkin method it does not rely on information that is unavailable at the time of expectation formation, and allows for meaningful tests of expectation characteristics like unbiasedness. 
When working with quantified expectations it is always sensible to assess the robustness of the hypotheses with different quantification methods since the actual expectation formation process of survey respondents is unknown. The method proposed in this paper can be used for such analyses.

Acknowledgements. The author would like to thank the anonymous referee for his valuable comments and suggestions that helped to improve the quality of this paper.

\section{References}

Anderson, O. (1952) The business test of the IFO Institute for Economic Research, Munich, and its theoretical model, Review of the International Statistical Institute, 20, 1-17.

Carlson, J.A. and Parkin, M. (1975) Inflation expectations, Economica, 42, 123-138.

Henzel, S. and Wollmershäuser, T. (2005) Quantifying Inflation expectations with the CarlsonParkin method: A survey-based determination of the just noticeable difference, Journal of Business Cycle Measurement and Analysis, 2, 321-352.

Madeira, C. and Zafar, B. (2014) Heterogeneous inflation expectations and learning, Journal of Money, Credit and Banking, 47, 867-896.

Malmedier, U. and Nagel, S. (2015) Learning from inflation experiences, Quarterly Journal of Economics, first published online October 1, 2015.

Pesaran, M. (1984) Expectations formation and macroeconomic modelling. Contemporary macroeconomic modelling (27-55), Blackwell, Oxford.

Theil, H. (1952) On the shape of economic microvariables and the Munich Business Test. Révue de l'Institut International de Statistique, 20, 105-120. 